AperTO - Archivio Istituzionale Open Access dell'Università di Torino

Favorable short-term outcome of hepatitis C virus-positive liver graft with bridging fibrosis: A plea for very early viral eradication

This is a pre print version of the following article:

Original Citation:

Availability:

This version is available http://hdl.handle.net/2318/1627479 since 2017-06-08T00:14:10Z

Published version:

DOI:10.1002/hep.28978

Terms of use:

Open Access

Anyone can freely access the full text of works made available as "Open Access". Works made available under a Creative Commons license can be used according to the terms and conditions of said license. Use of all other works requires consent of the right holder (author or publisher) if not exempted from copyright protection by the applicable law. 


\section{HEPATOLOGY}

\section{Favorable short-term outcome of HCV-positive liver graft with bridging fibrosis: a plea for very early viral eradication}

\begin{tabular}{|c|c|}
\hline Journal: & Hepatology \\
\hline Manuscript ID & HEP-16-1786.R1 \\
\hline Wiley - Manuscript type: & Clinical Observations in Hepatology \\
\hline Date Submitted by the Author: & 24-Oct-2016 \\
\hline Complete List of Authors: & $\begin{array}{l}\text { Martini, Silvia; AOU Città della Salute e della Scienza di Torino, University } \\
\text { of Torino, Gastrohepatology Unit } \\
\text { Salizzoni, Mauro; AOU Città della Salute e della Scienza di Torino, } \\
\text { University of Torino, Liver Transplantation Center and General Surgery } 2 U \\
\text { David, Ezio; AOU Città della Salute e della Scienza di Torino, University of } \\
\text { Torino, Pathology Unit } \\
\text { Tandoi, Francesco; AOU Città della Salute e della Scienza di Torino, } \\
\text { University of Torino, Liver Transplantation Center and General Surgery } 2 U \\
\text { Fonio, Paolo; AOU Città della Salute e della Scienza di Torino, University of } \\
\text { Torino, Radiology Unit } \\
\text { Delsedime, Luisa; AOU Città della Salute e della Scienza di Torino, } \\
\text { University of Torino, Pathology Unit } \\
\text { Strona, Silvia; AOU Città della Salute e della Scienza di Torino, University } \\
\text { of Torino, Gastrohepatology Unit } \\
\text { Dell Olio, Dominic; AOU Città della Salute e della Scienza di Torino, } \\
\text { Regional Transplantation Center, Piedmont } \\
\text { Saracco, Giorgio; AOU Città della Salute e della Scienza di Torino, } \\
\text { University of Torino, Gastrohepatology Unit } \\
\text { Romagnoli, Renato; AOU Città della Salute e della Scienza di Torino, } \\
\text { University of Torino, Liver Transplantation Center and General Surgery } 2 U\end{array}$ \\
\hline Keywords: & $\begin{array}{l}\text { Organ shortage, Liver fibrosis, Direct-acting antiviral agents, HCV-positive } \\
\text { donor liver }\end{array}$ \\
\hline
\end{tabular}




\title{
Favorable short-term outcome of HCV-positive liver graft with bridging fibrosis: a plea for very early viral eradication
}

\author{
Silvia Martini ${ }^{1}$, Mauro Salizzoni ${ }^{2}$, Ezio David ${ }^{3}$, Francesco Tandoi $^{2}$, \\ Paolo Fonio ${ }^{4}$, Luisa Delsedime ${ }^{3}$, Silvia Strona ${ }^{1}$, Dominic Dell Olio ${ }^{5}$, \\ Giorgio Maria Saracco ${ }^{1}$, Renato Romagnoli ${ }^{2}$ \\ ${ }^{1}$ Gastrohepatology Unit \\ ${ }^{2}$ Liver Transplantation Center and General Surgery $2 \mathrm{U}$ \\ ${ }^{3}$ Pathology Unit \\ ${ }^{4}$ Radiology Unit \\ ${ }^{5}$ Regional Transplantation Center, Piedmont
}

AOU Città della Salute e della Scienza di Torino, University of Torino, Italy

\author{
Authors' e-mail \\ Silvia Martini: smartini@cittadellasalute.to.it \\ Mauro Salizzoni: mauro.salizzoni@unito.it \\ Ezio David: ezio.david@unito.it \\ Francesco Tandoi: francesco.tandoi@gmail.com \\ Paolo Fonio: paolo.fonio@unito.it \\ Luisa Delsedime: Idelsedime@cittadellasalute.to.it \\ Silvia Strona: silvia.strona@gmail.com \\ Dominic Dell Olio: dellolio@hotmail.com \\ Giorgio Maria Saracco: giorgiomaria.saracco@unito.it \\ Renato Romagnoli: renato.romagnoli@unito.it
}




\section{Keywords}

Organ shortage; HCV-positive donor liver; liver fibrosis; direct-acting antiviral agents.

\section{Contact Information}

Silvia MARTINI, MD

Gastrohepatology Unit, AOU Città della Salute e della Scienza di Torino

Corso Bramante 88, 10126 Turin, Italy

Phone: +39011 6335928; Fax: +390116334014

E-mail: smartini@cittadellasalute.to.it

\section{List of Abbreviations}

LT, liver transplantation; HCV, hepatitis C virus; MELD, Model for End-stage Liver Disease; CIT, cold ischemia time; DAA, direct-acting antiviral agents; US, ultrasound; GGT, gammaglutamyltransferase; HBV, hepatitis B virus; SVR12, sustained virological response at week 12 after end of therapy; kPa, kilopascal.

\section{Financial Support}

The authors did not receive any grant or financial support for this work. 
2

3

4

\section{Summary}

We report the first case of an HCV-positive recipient who was transplanted with an HCVpositive graft with portal-portal bridging fibrosis. Curbing HCV infection immediately after liver transplant with direct-acting antivirals, resulted in an excellent 9-month outcome. 
Persistent organ shortage in liver transplantation (LT) forces transplant centers to use extended criteria donors. In this setting, hepatitis C virus (HCV)-positive livers represent a precious resource, but their use has been so far restricted to those with fibrosis stage $\leq 2$ according to Ishak (i.e. absence of bridging fibrosis), pending studies on fibrosis progression in grafts at more advanced stage (1). The same indication accrued from our own experience(2). In 1998 we used a 45-year-old HCV-positive graft having bridging fibrosis (staging 3/6, Ishak) in an HCV-positive recipient, Model for End-stage Liver Disease (MELD) 19 at LT; cold ischemia time (CIT) was 689 minutes and the patient experienced delayed non-function (prolonged ascites with progressive cholestasis), requiring re-LT on day 75 .

However, the recent advent of direct-acting antivirals (DAA) has revolutionized HCV therapy (32) and changes in the outcome of HCV-positive grafts can be expected. On December $10^{\text {th }}, 2015$, the $2771^{\text {th }}$ LT was performed in Turin, Italy. The donor was a 46year-old male, deceased of anoxic brain injury. He was HCV positive, genotype 3a, inexperienced to antiviral therapy. Abdomen ultrasound (US) showed normal liver and spleen echogenicity and size. Platelet count was $229 \times 10^{9} / \mathrm{L}$, total bilirubin $0.7 \mathrm{mg} / \mathrm{dL}$, INR 1.1, AST/ALT 98/171 IU/L, gamma-glutamyltransferase (GGT) 297 IU/L, HCV-RNA $1,210,101 \mathrm{IU} / \mathrm{mL}$, hepatitis B virus (HBV) core antibody positive. On retrieval, the liver was soft despite a grainy capsule (Fig.1A); biopsy showed fibrous expansion of most portal areas with evidence of portal-portal bridging (grading 4/18, staging 3/6, Ishak) (Fig.1B-C). The recipient, who signed an informed consent, was a 60-year old male, affected by HBV/HCV-related cirrhosis (HCV genotype 1b, non-responder to peginterferon/ribavirin), portal hypertension (large esophageal varices, spleen diameter $20 \mathrm{~cm}$ ) and multinodular hepatocellular carcinoma beyond San Francisco criteria, successfully downstaged to Milan criteria. At LT, his MELD score was 8, creatinine clearance 98 mL/min, HCV-RNA 150,656 
IU/mL; HBV-DNA undetectable without treatment. CIT was 373 minutes; end-to-end biliary anastomosis with T-tube was performed; post-reperfusion biopsy showed moderate ischemia-reperfusion injury. Immediate graft function was satisfactory, with no ascites and no evidence of early/delayed dysfunction. Post-LT course was complicated by pneumonia due to a multi-sensitive Klebsiella pneumonize, and he was discharged on day 26. Immunosuppression included tacrolimus, mycophenolate mofetil and steroids, the last weaned within 6 months. He underwent anti-HBV prophylaxis with lamivudine and hepatitis B immunoglobulin. Anti-HCV therapy with daily sofosbuvir (400 mg), daclatasvir $(60 \mathrm{mg})$ and ribavirin at escalating dose (from 400 to $1000 \mathrm{mg}$ ) was started on the day of transplant for 24 weeks (according to Italian guidelines), with good tolerance. After LT, HCV-RNA was 4,407 IU/mL (HCV genotype 3a) at week $1 ;<15 \mathrm{IU} / \mathrm{mL}$ at week 2 and persistently undetectable since week 3 . He achieved a sustained virological response at week 12 after end of therapy (SVR12). Transaminases and total bilirubin were persistently normal from week 4 after LT; GGT and alkaline phosphatase normalized from week 8.

Liver stiffness, evaluated by transient elastography was 10.5 kilopascal $(\mathrm{kPa})$ at week 12 of therapy and it fell to $8.5 \mathrm{kPa}$ at SVR12.

On May $2^{\text {nd }}, 2016$, a T-tube cholangiography showed a mild biliary anastomotic stricture, which was managed by endoscopic stenting for 1 month.

At SVR12, Doppler-US showed regular liver perfusion and reduction in spleen size (16.5 $\mathrm{cm}$ ), whilst upper endoscopy confirmed disappearance of the esophageal varices; magnetic resonance cholangiopancreatography evidenced a patent biliary anastomosis with normal intrahepatic bile ducts (Fig.2A); liver biopsy showed persistent fibrous expansion of most portal areas with occasional portal-portal bridging (grading 3/18, staging 3/6, Ishak; Fig.2B-C).

In conclusion, recourse to well-tolerated, pangenotipiepan-genotypic DAAs immediately after LT, allowed to cure the donor's chronic hepatitis $C$ into the recipient, thus affording a 
favorable short-term outcome, at variance with our previous unsuccessful case occurring in the interferon-era. The present report is a proof-of-concept that curbing HCV infection very early after LT, in association with CIT minimization and careful recipient selection, may result in excellent survival of HCV-positive grafts with portal-portal bridging, pending long-term fibrosis evaluation. This observation should encourage transplant teams to a more extensive use of HCV-positive donors, hopefully within the scope of an internationally agreed multicenter protocol.

\section{References}

1. Coilly A, Samuel D. Pros and cons: usage of organs from donors infected with hepatitis $\mathrm{C}$ virus $\underline{\mathrm{HCV}}$. Revision in the direct-acting antiviral era. J Hepatol 2016;64:226-231.

2. Ricchiuti A, Brunati A, Mirabella S, Pierini A, Franchello A, Salizzoni M. Use of hepatitis $G$ virus-positive grafts in liver transplantation: a single-centre experience. Transplant Proc. 2005;37:2569-2570.

3. 2. Hepatitis C guidance: AASLD-IDSA recommendations for testing, managing, and treating adults infected with hepatitis C virus $\underline{\mathrm{HCV}}$. Hepatology 2015;62:932-954. 


\section{FIGURE LEGENDS}

Figure 1. Macroscopic and microscopic features of HCV-positive donor liver on retrieval. (A) Macroscopic appearance of the liver on retrieval: despite a grainy Glisson capsule, the parenchymal texture was soft on palpation, and no signs of portal hypertension were visible and spleen size was normal. (B) Portal inflammation with lymphocytic aggregate (empty arrow) and periportal hepatitis (arrows); grading 4/18 according to Ishak score (Sirius Red staining; x 200 magnification). (C) Portal fibrosis (empty arrow) with portal-portal bridging (arrows) (staging 3/6 according to Ishak score) and diffuse mild macrovesicular and microvesicular steatosis (Masson's Trichrome staining, which was performed as an urgent procedure using rapid technique; $\times 100$ magnification).

Figure 2. Biliary imaging and histology at 9-month follow-up after transplantation and subsequent very early viral eradication. (A) Magnetic resonance cholangiopancreatography showing normal intrahepatic bile ducts, patent biliary anastomosis (arrow) and mild dilatation of the extrahepatic bile duct. (B) Mild portal inflammation (arrow), focal periportal hepatitis (arrowhead) and focal lobular inflammation (empty arrow); grading 3/18 according to Ishak score (hematoxylin-eosin staining; $x 100$ magnification). (C) Portal fibrosis (arrow) and thin portal-portal bridging (empty arrow); staging 3/6 according to Ishak score (Sirius Red staining; x 100 magnification). 

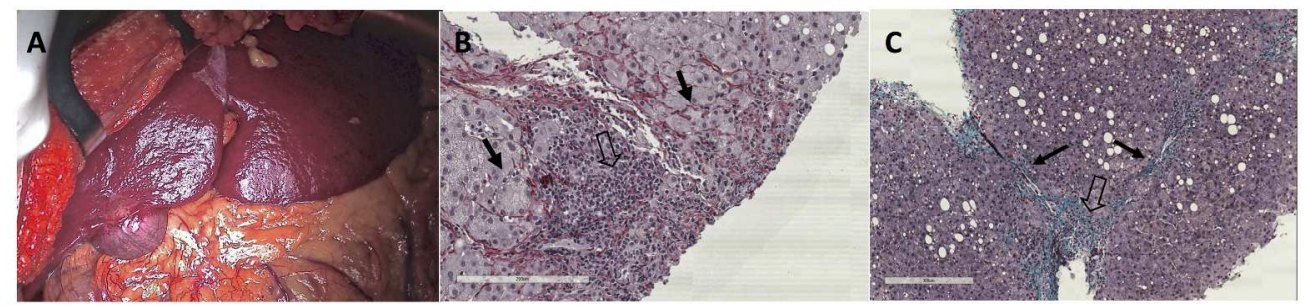

Figure 1. Macroscopic and microscopic features of HCV-positive donor liver on retrieval. (A) Macroscopic appearance of the liver on retrieval: despite a grainy Glisson capsule, the parenchymal texture was soft on palpation, no signs of portal hypertension were visible and spleen size was normal. (B) Portal inflammation with lymphocytic aggregate (empty arrow) and periportal hepatitis (arrows); grading 4/18 according to Ishak score (Sirius Red staining; x 200 magnification). (C) Portal fibrosis (empty arrow) with portal-portal bridging (arrows) (staging 3/6 according to Ishak score) and diffuse mild macrovesicular and microvesicular steatosis (Masson's Trichrome staining, which was performed as an urgent procedure using rapid technique; x 100 magnification).

$253 \times 67 \mathrm{~mm}(300 \times 300$ DPI $)$ 


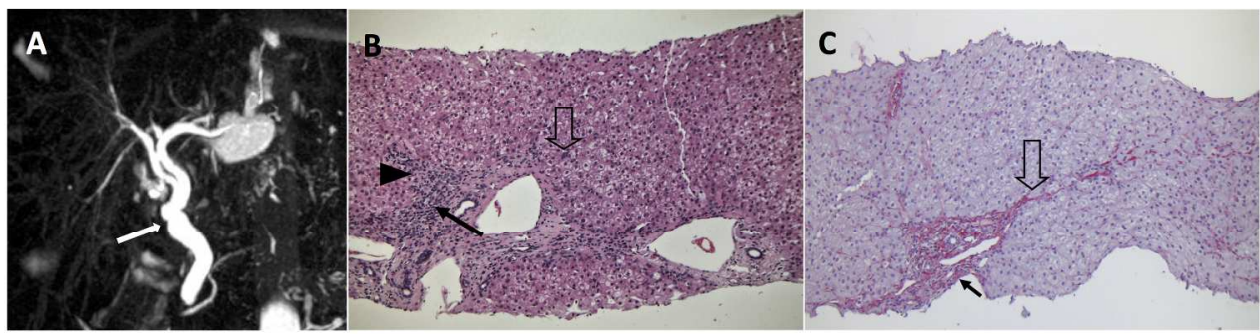

Figure 2. Biliary imaging and histology at 9-month follow-up after transplantation and subsequent very early viral eradication. (A) Magnetic resonance cholangiopancreatography showing normal intrahepatic bile ducts, patent biliary anastomosis (arrow) and mild dilatation of the extrahepatic bile duct. (B) Mild portal inflammation (arrow), focal periportal hepatitis (arrowhead) and focal lobular inflammation (empty arrow); grading 3/18 according to Ishak score (hematoxylin-eosin staining; x 100 magnification). (C) Portal fibrosis (arrow) and thin portal-portal bridging (empty arrow); staging 3/6 according to Ishak score (Sirius Red staining; x 100 magnification).

$238 \times 68 \mathrm{~mm}(300 \times 300 \mathrm{DPI})$ 